\section{NEST: A program to verify proper RATFOR nesting structure}

\author{
ARTHUR D. FISK \\ AT\&T Long Lines, Cincinnati, Ohio 45202 \\ and \\ WALTER SCHNEIDER \\ Human Attention Research Laboratory \\ University of Mlinois, Champaign, Mlinois 61820
}

The NEST program provides the user with a means of checking for nesting errors in RATFOR programs. It gives users the ability to verify not only the normal open and closed bracket symbols, but, more important, their own bracket-defined symbols (such as ENDIF for closed bracket).

RATFOR is a structured language that is available on many computers (e.g., IBM, Cyber, DEC PDP-11, Apple) and is easily transportable among computers. RATFOR is a preprocessor that requires a FORTRAN compiler. A useful feature of RATFOR is its DEFINE statement which allows the programmer to define one alphanumeric string to replace another alphanumeric string. An example of the use of the DEFINE feature is to define the character string BEGINLOOP to function as an open bracket. This feature increases the readability of the RATFOR code and, in general, decreases debugging time. However, the RATFOR preprocessor does not provide a means for verifying the proper nesting structure (i.e., proper use of open and closed brackets).

We have found that debugging time can be reduced when programmers are able to check their program's nesting structure via a mechanized tool. The program described here provides such a mechanized tool. The program allows the user to specify a file of user-defined bracket alphanumeric strings (the file can also be used in a RATFOR INCLUDE statement). The program then checks a specified RATFOR program for proper nesting based upon the user-defined brackets. Of course, the predefined RATFOR open and closed brackets are also verified by the program.

Input. The user is prompted to enter the name of the RATFOR program that is to be checked and the file containing the definition for open and closed brackets. The user may request that all text or only the description of the nesting be output.

Output. On a given line of output, the program presents the nesting level (a numeric) followed by the bracket symbol encountered, the line number in the program, and, finally, a symbolic representation of the nesting level. An example of an output line is:

\section{LOOP $97 \quad * * * * * * *$}

This implies that on Line 97 of the program, a "LOOP" structure was encountered and now the program is at the seventh level of nesting. The ******* visually represents the level of nesting. For each program scanned, a header is output for ease of understanding the output lines.

If inappropriate nesting is encountered, the program indicates the error and outputs the invalid nesting symbol. An example of this error output is: "ERROR-Nesting is negative, encountered an extra ENDLOOP in line \#29."

If the "all text" option is used, all program lines are printed to the right of the nesting description (up to 120 characters/line).

The program is useful because of the speed with which code can be checked (speed is primarily limited by the output device). We have found that nesting errors often are the result of logic errors, and the present program is a means to quickly verify that program bugs are not the result of such errors.

Computers and Availability. The program is written in RATFOR for both DEC RT-11 and UNIX operating systems, and requires approximately $5 \mathrm{~KB}$ words.

Program listings and a sample run (input and output) are available from the first author. Please specify whether the RT-11 or UNIX version is desired.

(Accepted for publication October 16, 1982.) 